Revista Digital Año 9. No 12 - Año 2018. -pág. 1--110

ISSN 1853-1393

Resistencia. Chaco. Argentina - 2018

\title{
BUENAS PRÁCTICAS DOCENTES PARA LA INCLUSIÓN EDUCATIVA DE LA PRIMERA INFANCIA EN LA ESCUELA DE GESTIÓN SOCIAL DEL BARRIO SEGUNDO DAVID PERALTA "MATE COSIDO", RESISTENCIA, CHACO
}

GOOD TEACHING PRACTICES FOR THE EDUCATIONAL INCLUSION OF EARLY CHILDHOOD IN THE SOCIAL MANAGEMENT SCHOOL, CALLED SEGUNDO DAVID PERALTA, KNOWN TOO AS “MATE COSIDO", IN RESISTENCIA, CHACO PROVINCE, ARGENTINA

\author{
Norma Elena Bregagnolo ${ }^{1}$ - Zulema del Carmen Nussbaum² ${ }^{2}$ Marina Stein $^{3}$
}

Fecha de recepción: 09-11-2017

Fecha de aceptación y versión final: 28-03-2018

Resumen: Esta comunicación se realiza en el marco de la investigación "Buenas Prácticas en la Educación Infantil. Estudio de casos en el Área Metropolitana del Gran Resistencia- Chaco" -(UNNE), PI H007 -, aprobado por Resolución № 984/14 - C.S. UNNE, enmarcada en el Proyecto Internacional "Diseño Curricular y Buenas Prácticas en la Educación Infantil: una visión internacional, multicultural e interdisciplinar" dirigido por el Dr. Zabalza.

Es un estudio de tipo exploratorio, aborda prácticas pedagógicas realizadas en el Jardín Maternal y de Infantes de la Escuela Pública de Gestión Social № 1 "Héroes Latinoamericanos", cuya creación fue impulsada por el Movimiento Político - Social denominado Movimiento Territorial de Liberación - MTL - . Entre sus objetivos se propone describir las concepciones docentes en torno a las "Buenas Prácticas" en la Educación Infantil e identificar, analizar y visibilizar casos de "Buenas Prácticas" en contextos particulares.

Para la obtención de información se realizaron entrevistas en profundidad, observación de clases y análisis documental.

Se visualiza en el recorrido de este trabajo como desde los diferentes actores de la institución se generan permanentemente dinámicas de participación y trabajo conjunto con las familias, y a la vez propuestas integrando a toda la institución.

\footnotetext{
${ }^{1}$ Profesora en Jardines de Infantes y Especialista en Jardines Maternales - UNNE - Licenciada en Educación Inicial - UnaF - Especialista en Docencia Universitaria - Diplomada en Extensión Universitaria - REXUNI - UNGS - Profesora Adjunta Taller de Integración, Investigación y Práctica I - Prof. y Lic. en Educación Inicial - Secretaria de Extensión, Capacitación y Servicios - Facultad Humanidades - UNNE Sub Directora del equipo de investigación del Proyecto "Buenas Prácticas en la Educación Infantil. Estudio de casos en el Area Metropolitana del Gran Resistencia- Chaco". Investigadora Cat. IV Domicilio: 1o de Mayo № 775 - Resistencia, Chaco - Teléfono móvil: 3624384509. Dirección de correo: normabregagnolo@yahoo.com.ar

${ }_{2}^{2}$ Profesora en Educación Pre-elemental. Prof. en Ciencias de la Educación. Especialista en Docencia Universitaria. Especialista en Educación Maternal. Diplomada en Educación, Infancia y Pedagogía. Profesora Titular del Taller Administración Educativa y Profesora Adjunta del Taller de Integración, Investigación y Práctica I. Integrante del equipo de investigación del Proyecto "Buenas Prácticas en la Educación Infantil. Estudio de casos en el Area Metropolitana del Gran Resistencia- Chaco". San Lorenzo 1015 - Resistencia, Chaco -, Teléfono móvil: 3624-069267. Dirección de correo: zulema cn@hotmail.com

${ }^{3}$ Doctora en Biología - Profesora Adjunta de Educación para la Salud en la Primera Infancia del Profesorado y Licenciatura en Educación Inicial - Facultad de Humanidades - UNNE - Integrante del equipo de investigación del Proyecto "Buenas Prácticas en la Educación Infantil. Estudio de casos en el Area Metropolitana del Gran Resistencia- Chaco"- Investigadora Categoría III. Domicilio: Avda. Las Heras № 2664 - Resistencia, Chaco -, Teléfono móvil: 3624659806. Dirección de correo: marinastein66@gmail.com
} 
Palabras clave: Primera Infancia - Inclusión - Buenas prácticas - Derechos del Niño - Gestión comunitaria

Abstract: This communication is made in the framework of the research "Good Practices in Childhood Education. A case study in the Greater Resistencia-Chaco Metropolitan Area "- (UNNE), PI H007 -, by Resolution No. 984/14 - C.S. UNNE, gramed in the International Project "Curricular Design and Good Practices in Early Childhood Education: an international, multicultural and interdisciplinary vision" directed by Dr. Zabalza.

It is an exploratory study, approaches pedagogical practices carried out in the Maternal and Infants Garden of the Public School of Social Management No. 1 "Latin American Heroes", whose creation was promoted by the Political - Social Movement called Territorial Movement of Liberation - MTL - .

Among its aims, it is proposed to describe the teaching conceptions around the "Good Practices" in Childhood Education and to identify, analyze and make visible "Good Practices" in particular contexts.

In the course of this work, it is visualized as from the different actors of this institution how they generate dynamics of participation and collaborative work with families permanently, and at the same time proposals for integration to the whole institution.

Key words: Early childhood-inclusion-good practices-children's rights-community management 


\section{Introducción}

Este trabajo de investigación se realizó en el Jardín Maternal y de Infantes de la primer Escuela de Gestión Social $N^{\circ}$ 1"Héroes Latinoamericanos", del gran Resistencia. A partir del estudio exploratorio realizado se pretende indagar las concepciones de Ixs docentes sobre buenas prácticas en Educación Infantil. En esta oportunidad intentamos mostrar un avance de lo realizado hasta el momento. Dicha Escuela es de Gestión Social, la misma fue creada en el 2011, y se ubica dentro de la educación formal, estatal y pública.

El Ministerio de Educación, Cultura, Ciencia y Tecnología en la provincia del Chaco, crea las instituciones educativas de dos maneras. Una de ellas tiene que ver con dar respuesta a reclamos o demandas que la sociedad le hace llegar o bien desde la prospectiva de quienes diseñan, planifican y ejecutan los grupos habitacionales.

En este caso en particular, esta escuela surge del reclamo del Movimiento Territorial de Liberación (MTL). Una de sus referentes, Cristina Canteros, destaca que se origina "de una concepción de generación del hombre nuevo por lo que se hace necesario empezar desde el jardín, pensar en un hombre nuevo absolutamente solidario, ese es uno de los principales valores que se quiere enseñar". Uno de los objetivos fundamentales de este proyecto es la efectivización del derecho a la educación de Ixs niñxs del Barrio. En respuesta a los reclamos realizados y teniendo en cuenta las políticas de educación pública desde el M.E.C.C. y T. se crea la Escuela Pública de Gestión Social E.P.G.S. N 1 por Resolución N ำ 2746/12.

Actualmente a esta institución asisten 170 niños, entre 45 días y 5 años, con un plantel docente integrado por una directora y 10 docentes de Educación Inicial y profesores de: Educación Física, Huerta escolar e inglés. Acompañan como auxiliares y personal de servicios mujeres, madres, cuyos hijos asisten al jardín y que viven en el barrio e integran el Movimiento Territorial de Liberación (MTL).

"Comenzamos en el 2005 con el anexo, y luego los docentes que venían a este espacio no eran los docentes adecuados formados para estos espacios altamente vulnerables, coincidíamos con los docentes en que es una población altamente vulnerable. Comenzamos a gestionar como escuela independiente, la E.G.S. El Barrio cumplió 15 años" ( Cantero',C.,2017)

La escuela tiene un lugar de mucha importancia para el movimiento, ya que de esta manera la población considera que no solo estarían luchando por el derecho a la educación para sus hijos, sino que además y el mayor desafío en la efectivización de este derecho para Ixs niñxs es que ella pueda brindar las más variadas y mejores oportunidades educativas a estxs niñxs, "muchas veces no es visibilizado por los padres esta importancia" (G. docente de la EGS N¹).

La mejora de la calidad de la educación infantil es, sin duda, un proceso complejo y en el que intervienen muchos factores: desde las políticas educativas hasta los recursos disponibles; desde las particulares tradiciones y culturas de cada país hasta los sistemas de financiación y organización de los dispositivos dedicados a la infancia en cada momento; desde la formación y experiencia del profesorado hasta la capacitación y la particular percepción de las familias sobre la educación temprana. Pero de todos ellos, el factor que ejerce una influencia más determinante en la calidad de la educación infantil y, a su vez, el más sujeto a iniciativas de mejora es, sin duda, la actividad de los profesionales y las prácticas educativas que estos desarrollan, como en el caso de la Escuela de Gestión Social №1 y en particular el Jardín Maternal y de Infantes. En este sentido "Tal vez no sea del todo pedagógico, pero debemos dar desde el sentimiento de amor, abrazo, desde el amor para romper con esa barrera, la del dolor, de la exclusión." Señala con convicción (énfasis) Cristina Cantero 2017. 


\section{Marco teórico}

El denominado movimiento piquetero surge en Argentina a finales de la década de 1990 como expresión de una multiplicidad de organizaciones de desocupados cuya reivindicación central era la generación de puestos de trabajo y cuya metodología principal era la interrupción del tránsito vehicular en calles y rutas.(Román, M."i)

Las políticas neoliberales de los $90 `$ cambiaron el paisaje económico, político y social en Argentina. Como consecuencia muchos agricultores y pobladores de medianas y pequeñas localidades de la provincia de Chaco se vieron obligados a emigrar a las principales ciudades (Carli, 2002: 16). En Resistencia, capital de la provincia, también la emigración de las poblaciones en busca de una mejora hizo que las zonas periféricas de la ciudad se vieran de pronto, ocupadas por los denominados "asentamientos", donde en su mayoría, en terrenos del estado, libre (en el sentido de no tener ningún tipo de construcción) se transformaron en una posibilidad de mejora para estas personas. Este mismo movimiento se produjo desde el centro de la ciudad por familias que no podían pagar los alquileres de sus casas o quedaron sin trabajo y debieron venderlas.

Esta política generó una pobreza estructural y propició la exclusión de aquellos grupos más vulnerables de la población, "quienes, según Tapia (2009), debieron asumir sus conquistas de supervivencia desde las luchas y organizaciones sociales", frente a tantas necesidades que influyeron y repercutieron en la manera de asumir y convivir entre todos los actores sociales pero particularmente con la primera infancia, donde la escases, las luchas, resistencias y las privaciones se transformaron en elementos recurrentes. Tal lo expresa Tapia (2009), "un movimiento social empieza a configurarse cuando la acción colectiva empieza a desbordar los lugares estables de la política, tanto en el seno de la sociedad civil como en el estado y se mueve a través de la sociedad buscando solidaridades y aliados en torno a un cuestionamiento sobre los criterios y formas de distribución de la riqueza social o de los propios principios de organización de la sociedad, del estado y del gobierno".

Estos cambios, generaron, fundamentalmente, un movimiento que promovía y promueve la autonomía en la interacción social. Se trata de un proceso que se llevó a cabo en una comunidad determinada y que se basó en el aprendizaje colectivo, continuo y abierto para el diseño y la ejecución de proyectos que atendieran necesidades y problemas sociales. La gestión social implicó el diálogo entre diversos actores, donde la autogestión, desarrollo comunitario o gestión comunitaria se transformaron en componentes relevantes. La autogestión generó formas de autoorganización, de autogobierno y en ocasiones de autogestión económica, nutriéndose de las realidades, sus problemas y conflictos sociales, promoviendo la incorporación de muchos sujetos alrededor de sus necesidades y valores, lo cual propició posibilidades de participación comprometida, activa y lúcida de los sujetos, que favorecieron los "cambios de abajo para arriba" (Tapia, 2009:4).

En el caso de la provincia del Chaco, en el nordeste argentino, las primeras organizaciones de desocupados surgieron de una interrelación de factores entre los cuales se destacan: las protestas por la inundación en barrios periféricos de la capital, la existencia de una masa de población desocupada producto de la temprana descomposición económica de la provincia y la intervención de militantes de diferentes tendencias políticas con experiencia en el trabajo sindical, vecinal o partidario. Todos estos factores confluyeron hacia 1999 en la creación de la primera organización de desocupados con permanencia temporal y visibilidad pública: el Movimiento de Trabajadores Desocupados (MTD) General San Martín (Román, M.)

Es así y cómo a raíz de estos cambios en la sociedad surge en el campo de lo educativo la Escuela de Gestión Social. 
"Esta es brindada por organizaciones sin fines de lucro, a través de una pedagogía popular, cooperativa y solidaria para el logro de una ciudadanía crítica y emancipada. Responde a acciones y valores que definen las construcciones colectivas de democracia participativa, donde el cooperativismo es herramienta para la socialización del conocimiento, que aborda además las necesidades de la comunidad en que está inserta, que se caracteriza por atender poblaciones pobres, de familias desescolarizadas que provienen de migraciones internas." (Oporto, M.L.: 2015, 25).

La Organización de Estados Iberoamericanos (OEI) expresa en su documento sobre "Sistema de Información sobre Primera Infancia en Iberoamérica. Documento de presentación" (2009: pág.15 y 16) lo siguiente: se concibe que es el Estado el responsable de garantizar las condiciones necesarias para que todos los individuos, por igual, puedan gozar y reivindicar el cumplimiento de los derechos de los cuales son titulares. Desde este enfoque Ixs niñxs son considerados sujetos de derechos y sus derechos son considerados derechos humanos, son interdependientes $e$ indivisibles porque cada uno aporta un significado adicional a los demás. Pensarlos desde otro lugar implica la no efectivizaciòn desde su sentido pleno de los mismos y por lo tanto vulnerar los derechos de los que son beneficiarios Ixs niñxs.

Es por lo que en el mencionado texto se considera que los Estados deben garantizar el cumplimento de todos los derechos y a todos. En el contexto Argentino de los 90' "marcado por el desempleo, la movilidad descendente y el aumento de la pobreza produjo una brecha mayor entre generaciones contemporáneas en cuanto a condiciones de vida y horizontes de futuro y un aumento notorio de la desigualdad social dentro de la misma generación infantil" (Carli 2006: 20). Actualmente se mantiene esa desigualdad, si bien el Estado visibiliza al/la niñx como sujeto de derecho, la "sociedad infantil" se presenta con marcadas diferencias sociales, por un lado, niñxs con acceso a las más modernas tecnologías en el resguardo del hogar y por otro niñxs que reemplazan los tiempos de juego y educación, transitando por las calles buscando cómo sobrevivir.

Menciona el texto de la OEI que, (...) "el cumplimiento de los derechos no puede asegurarse tan solo observando si los Estados cumplen con algunos de los intereses tutelados por un derecho social sino desentrañando si la población de ese país puede invocar ese derecho, en caso de incumplimiento para establecer un reclamo, queja o demanda que dé lugar al dictado de una sentencia que imponga el cumplimiento de la obligación que le es debida. En esto consiste la verdadera asistencia de un sujeto de derecho; si no existe demandabilidad o exigibilidad, no existe derecho pleno y el titular del mismo no es sujeto de derecho sino de una gracia estatal."

La Convención sobre los Derechos del Niño (UNICEF, 1989), (Art. 28 Inciso d y e) cuando habla de garantizar el Derecho a la Educación dice que es necesario ".... adoptar medidas para fomentar la asistencia regular a las escuelas y reducir las tasas de deserción “. En su Art. 29 (Inciso a) (UNICEF, 1989: 29) destaca que la educación deberá desarrollar la personalidad, las aptitudes y la capacidad mental y física del niño hasta el máximo de sus posibilidades.

En este sentido, la propuesta desde la Escuela Pública de Gestión Social es un proyecto de escuela "no graduada":

Resta ahora ver como los reclamos sociales y la efectivización del derecho a la educación, se van tejiendo para hacer una trama diferente a la habitual. Una trama que pueda ir construyendo desde la reflexión y el análisis crítico y continuo las prácticas y que estas transformaciones puedan ser el motor de buenas prácticas. 
En definitiva, el desafío está en encontrar, analizar buenas prácticas, pedagógicas e institucionales. Según Zabalza (2012), definir qué entendemos por "buena práctica" implica acuerdos.

Para Zabalza Beraza (2012), trabajar en la perspectiva de las "buenas prácticas" incluye, por tanto, tres procesos clave:

1) Justificar el sentido que tiene hablar de buenas prácticas y marcar las diferencias con respecto a otras modalidades de estudio de las prácticas reales en educación;

2) Identificar y analizar (tanto en su fundamentación como en su desarrollo efectivo) aquellas actuaciones que, por sus características y virtualidades, puedan categorizarse como "buenas prácticas";

3) Representar y visibilizar esas prácticas, de manera que puedan ser conocidas y transferidas a otras situaciones y actuar como puntos de referencia y/o contraste para quienes deseen avanzar en la mejora de la Educación.

Las investigaciones sobre desarrollo e innovación en instituciones educativas han ido poniendo de manifiesto que cualquier posibilidad de avance pasa por el compromiso del profesorado con los nuevos planteamientos de la calidad. Sin restar importancia a factores como la financiación y la disponibilidad de recursos, nada se conseguirá sin contar con los educadores y educadoras que son quienes han de convertir los nuevos retos y enfoques en actividades educativas concretas. También juegan un importante papel las familias pero, en su caso, la posibilidad de acceso y de influencia resulta más mediatizada por la propia privacidad de la vida familiar.

Es muy común encontrarse con voces que expresan que la práctica diaria es muy compleja, imprevista y rápida y que no da tiempo para hacer otro cosa. Como consecuencia y salvo importantes excepciones, la educación infantil se constituye en una acción profesional voluntariosa y muy comprometida pero con débiles fundamentos. Lxs educadorxs dedican mucha energía al desarrollo de las actividades planificadas pero no siempre existe un plan de actuación bien estructurado y fundamentado en evidencias de actuación. El trajín diario sobre el cual viven Ixs docentes tampoco genera espacios destinados a la reflexión y al análisis sobre sus propias prácticas, constituyendo muchas veces este tipo de prácticas habituales en recordatorio de anécdotas exitosas, más que en prácticas que impactan, y que generan reflexión y cambio.

El concepto de buenas prácticas, aunque de una semántica borrosa ha estado presente en el núcleo fundamental de la investigación educativa del último medio siglo. Incluso antes, pues como señala Van Haecht (1998), esa idea de ir a buscar referentes de buenas prácticas e instituciones modélicas al extranjero figuraba ya en escritos de inicios del S.XIX (L'Esquisse d'un ouvrage sur l'education comparée, de Jullien, 1816). Esta idea de buenas prácticas ha estado vinculada, con frecuencia, a otros referentes clásicos de la actividad educativa: innovación, actualización de recursos, calidad, evaluación, etc. A su vez, la idea de buenas prácticas, forma parte del repertorio de conceptos fluidos y flexibles con que los enfoques posmodernos se han enfrentado a las políticas sociales y educativas. Superada la idea de que pueda existir un modelo universal o una mejor forma de hacer que sea efectiva y transferible a cualquier situación, se ha buscado en la identificación de "buenas prácticas" aquellas modalidades diversas de responder, con eficacia y satisfacción de los participantes, a las diferentes demandas (en este caso educativas) del contexto (Benavente, 2007). En ese sentido, el concepto de buenas prácticas es aplicable a cualquier ámbito de la actividad humana, desde las políticas hasta las acciones específicas de sujetos individuales. (Proyecto: Buenas Prácticas en la Educación Infantil. Estudio de casos en el Área Metropolitana del Gran Resistencia- Chaco 2015).

Por último, otra cuestión que atraviesa a las buenas prácticas tiene que ver con la inclusión, entendida esta como la posibilidad del ingreso y la permanencia con 
calidad de los niños/as al sistema educativo formal. Hablar de calidad implica otorgar igualdad de posibilidades dentro del sistema educativo en contextos diferentes y muy difíciles o de gran vulnerabilidad para los niños y sus familias. Según Narodwsky (2008), "el mejoramiento en la calidad de la educación se desvanece a pesar de los enormes logros en materia de acceso y universalización de la educación básica y de inclusión en la agenda de quienes gobiernan los sistemas educativos; y de los problemas sociales que otrora estuvieran relegados, persisten fuertes desigualdades en materia de oportunidades educativas" (...) "El mejoramiento en la calidad se desvanece ante los problemas de acceso e inclusión, pues un sistema no ofrece educación de calidad, si no es razonablemente inclusivo".

Frente a todos estos elementos visibilizar, analizar y socializar buenas prácticas pedagógicas e institucionales se transforma más que otra cosa en un desafío que contemple la mirada crítica sobre lo fundamentalmente educativo sin dejar de lado todas aquellas otras cuestiones que atraviesan a las infancias y las marcan desde todos los lugares, de allí que el propósito del presente trabajo es indagar sobre las concepciones de Ixs docentes sobre buenas prácticas en Educación Infantil.

\section{Metodología de trabajo}

Durante el tiempo transcurrido en la implementación de este proyecto de investigación, que se articula con el Proyecto de Extensión "Infancia y Salud Integral: una propuesta educativa colaborativa con la Comunidad de la Escuela Pública de Gestión Social N ${ }^{\circ} 1$ "Héroes Latinoamericanos" que se viene realizando en el marco del Programa Universidad en el Medio - UNNE -, se ha trabajado en forma colaborativa a partir de entrevistas a informantes claves, observaciones de clases, registro de evidencias y análisis documental. También se realizaron registros fílmicos y fotográficos de actividades con familias y alumnxs, que servirán como insumo para trabajos posteriores.

Para este trabajo en particular se tomaron las entrevistas a informantes claves, a la directora del Jardín y a tres docentes.

Las entrevistas permiten reconocer lo que los protagonistas consideran como buenas prácticas pedagógicas y los casos o ejemplos que ilustran como buenas prácticas en la Escuela №1 de gestión comunitaria. Esta técnica de recolección de los datos sigue las pautas de una conversación entre iguales y esto es precisamente lo que permite una dinámica fluida y la profundización de diversos temas.

La triangulación de los datos permitió una mejor aproximación a la complejidad y multidimensionalidad del objeto de estudio.

\section{Las buenas prácticas de inclusión en la EGS № 1}

Los cambios vividos en el escenario político, social y económico en el país durante las últimas décadas han permitido la creación de novedosas maneras de participación y lucha. Estas últimas han generado nuevas formas de conquistas y efectivización de derechos para las y los niños.

Aquí cabría un interrogante al respecto: ¿De qué manera se logra el cumplimiento de los derechos para las nuevas generaciones si el Estado se corre? Sostenemos que estos nuevos formatos han sido una alternativa más de las que la sociedad se valió en un momento conflictivo. En relación con lo postulado por la Convención sobre los Derechos del Niño en cuanto a adoptar las medidas necesarias para fomentar la asistencia regular a las escuelas y reducir las tasas de deserción con el objeto de que la educación permita facilitar el desarrollo de la personalidad, las aptitudes y la capacidad mental y física del niño hasta el máximo de sus posibilidades 
puede mencionarse que la Escuela de Gestión Social es un nuevo formato dentro de la educación pública.

A través de esto el MTL ha podido materializar y efectivizar el derecho a la educación de las y los chicos en su entorno ya que ellos sostienen que a través del trabajo y la educación es posible tener una vida digna. En palabras de una de las fundadoras:

"el movimiento social y político que funda este barrio decide la construcción de una institución escolar para que cada uno de los habitantes se forme políticamente; trabajo, educación y salud es la forma de incluir y formarles (...) porque las patas para salir de este círculo de exclusión son la educación, el trabajo y la salud. Tenemos el barrio, tenemos la vivienda, tenemos trabajo, y con la escuela comenzamos desde el Nivel Inicial con la idea de formar al hombre nuevo, a la mujer nueva, entendemos que el camino es este, la educación." (Cantero,C., 2017).

Pero es sabido que la lucha por la existencia de la escuela es el primer escalón en la conquista realizada. Es necesario continuar e ir por lo que desde los principios del MTL se pretende y es lograr la efectivización también de buenas prácticas pedagógicas, que incluyan al contexto, las ideas y creencias que ellos tienen y que resulten en procesos de inclusión al sistema formal educativo de estos niñxs. Las buenas prácticas no pueden estar al margen de los contextos en las que ellas se implementan, es decir son buenas prácticas en contexto. Buenas prácticas porque responden a necesidades concretas de quienes participan en el proceso de aprendizaje, donde a decir de la fundadora del movimiento Cristina Canteros "buenas prácticas no deben ser prácticas estancas, sino requieren de pruebas y de resultados buenos, cuando algo no resulta se cambia; jhay que reinventar!"

Como un dato que nos permite saber desde que lugar estamos iniciando el proceso de inclusión se puede mencionar que en cuanto al contexto familiar de las y los niños que asisten al jardín Maternal y de Infantes de GS. № 1 puede mencionarse los siguientes datos recogidos a través del acompañamiento realizado por las docentes de la institución con relación a las y los niñas/os que asisten al Jardín maternal y de infantes. Dichos datos surgen de las entrevistas que realizan de manera domiciliarias a las familias las docentes del Jardín. Además, realizan visitas ante ausencias recurrentes de los niños reforzando esta idea de efectivizar el derecho a la educación de todos los/as niños/as, algo que ha ido generando cambios durante los últimos años. Estas ausencias ocasionadas por diferentes circunstancias se deben fundamentalmente según expresa la docente entrevistada: "a las ideas o concepciones de los tutores sobre los aportes de la escuela a la vida de sus hijos"(G.L). Es decir, las expectativas de que la educación pueda realmente servirles para su futuro.

Desde su creación a la fecha la matrícula fue en aumento, recibiendo niños/as de barrios vecinos, y de otras instituciones educativas. Pero ese aumento de matrícula en el nivel inicial no se continúa en la primaria donde la mayoría de los niños son anotados por los padres en las escuelas tradicionales.

De las entrevistas que las docentes realizan pudimos extraer la siguiente información del contexto familiar de los niños/as:

- Familias monoparentales (con ausencias de los padres o abandonos)

- Mujeres jefe de familia y que trabajan en diferentes oficios como: albañiles, electricistas y colaboran en los arreglos del Jardín Maternal y de Infantes o como auxiliares de las docentes en diferentes salas, cuyos ingresos económicos son solo del MTL

- Adicciones (droga y alcoholismo entre los detectados). 
- Hechos de violencia de género y abusos sexuales que sufren madres y niños (intrafamiliar). Otra información interesante que surge de las entrevistas tiene que ver con los objetivos que el equipo institucional se propuso: "acompañar y ser parte de la lucha por los cambios necesarios para transformar la realidad de las comunidades" y la necesaria empatía e identificación con los protagonistas de esos cambios, "no hacemos asistencialismo por eso hablamos con las familias y les contamos por qué los niños no almuerzan en el jardín, queremos que almuercen con sus familias", dice la directora, Prof. Lorena Ramírez. Adherimos al concepto de Perrone (2014) cuando dice:

"El acompañamiento pedagógico (que hoy llamamos "inclusión") requiere que los educadores aprendamos de los educandos. Requiere un notable esfuerzo de traducción mutua, para entender algo que la escuela moderna ignora o niega: que la condición de desarrollo del ser humano (...) es la comunidad. Y la comunidad tiene cultura, no espera a cursar la escuela o encender la televisión para proveerse de una. No la compra, la cultiva".

La referente del MTL agrega en este sentido:

"Si hablamos de inclusión es necesario practicar políticas de inclusión, por eso nuestra propuesta de escuela "no graduada", logra incluir a los jóvenes efectivamente. No existen punitivos como amonestaciones o quedar libres por faltas, se habla con los estudiantes, se les explica que deben cumplir con la aprobación de un programa para poder graduarse que requiere de su asistencia a la escuela".

Un aspecto novedoso de las prácticas docentes de la escuela de gestión social es la articulación entre jardín y primer grado, dónde en el primer grado participa durante la primera mitad del año una maestra de jardín trabajando conjuntamente con la maestra del primer grado de la escuela primaria. Este acompañamiento intenta trabajar sobre la noción de articulación propuesta en el Curriculum para la Educación Inicial Chaco (2012). La docente del Jardín acompaña a los niños y trabaja con la docente del primer grado de manera que para los niños el paso de un nivel al otro no resulte dificultoso.

En cuanto a la concepción de Buenas Prácticas coincidimos con la idea propuesta por Zabalza (2012: 22) quién señala que, de los principios pedagógicos convencionales, se pasa a una idea mucho más funcionalista y pragmática: dónde bueno es aquello que funciona bien, aquello que es valorado por sus protagonistas y beneficiarios, aquello que es reconocido como valioso por los colegas o por sus destinatarios indirectos. Creemos que estas prácticas expresadas como buenas por las docentes coincide con la idea de que diferentes contextos formativos requieren de actuaciones diversas. Si algo distingue los discursos sobre las "buenas prácticas", de otros más abstractos o desiderativos es que, en este caso, se habla de cosas que son, que se están haciendo, que han funcionado bien. En relación a esto la docente explica que trabajan con un proyecto Institucional denominado "educar para la paz" donde "trabajamos el compañerismo, la integración de todos los niños del jardín entre ellos y con todo el plantel docente". Esto surge porque muchos niños reproduciendo lo que dicen sus tutores los "discriminan" por ser hijos de padres que se alcoholizan o se drogan. "Se trata de no exponer a los niños en los conflictos de los padres, ni estigmatizarlos o etiquetarlos por su realidad diferente". Hablar de "calidad de vida" o "educación de calidad" en términos absolutos nos lleva a terrenos y consideraciones abstractas y difícilmente contrastables, por eso coincidimos en que debemos reducir esa consideración a elementos más tangibles y codificables (la calidad sustitutiva): los servicios médicos, los transportes públicos, los salarios, etc. aspectos faltantes en la 
realidad de estas familias. Las docentes aclaran que se trabaja teniendo en cuenta el curriculum, pero "muchas veces creemos más importante trabajar desde sus necesidades más inmediatas, como el caso del proyecto sobre Salud ambiental".

Puede recuperarse también un dato interesante realizado en este sentido, recogido de las entrevistas a la directora y que involucra una actividad que de manera sostenida y con un trasfondo de reclamo se viene realizando a través de una actividad integrada entre toda la comunidad educativa y con las familias de las y los niños que asisten a la EGS №1.

"El barrio está asentado en terrenos cercanos a lagunas de oxidación y hemos observado algunas reacciones en la piel en los niños. Hay olor desagradable proveniente de las lagunas que impregnan el aire. Lindero al Jardín y en un predio particularmente implicado con la educación se realizan actividades pedagógicas y lúdicas". (L. R. directora del Jardìn)

Por último, rescatamos la experiencia del campamento que organiza el profesor de educación física, que incluye actividades de campamento dónde se enseña a niños y padres a armar carpas, hacer fogatas y realizar distintos tipos de juegos con toda la familia. Se utiliza un espacio verde, amplio, de fácil acceso, ubicado frente a la institución, donde hace 3 años se ha proyectado y prometido desde el Ministerio de Educación Provincial la edificación de la escuela primaria y secundaria del barrio. El campamento es una "protesta" silenciosa que año a año reclama la edificación de esa escuela. En el mismo, se realizan juegos y construyen barriletes donde las diversas actividades se vuelven un desafío científico, formulan hipótesis, analizan los materiales utilizados, la velocidad del viento necesaria para que los barriletes puedan volar, entre otras actividades.

\section{A modo de cierre}

Como puede verse y en relación con lo que expresa Zabalza Beraza (2012) de los tres procesos clave podemos decir que hablar de buenas prácticas implica encontrar el sentido de lo que se está haciendo, ya que no se trata solo de lograr que Ixs niñxs estén en la escuela como fruto de políticas inclusivas, sino que además es necesario que en su permanencia puedan encontrar propuestas diferentes a lo que sus realidades complejas les brinda. Pero, para ello es necesario identificar y analizar aquellas actuaciones que puedan categorizarse como "buenas prácticas" y ¿cómo se llega a esta situación? Para ello es necesario encontrarlas, visibilizarlas de manera que puedan ser conocidas para luego ser transferidas a otras situaciones. Se evidencia en el recorrido de este trabajo como desde la institución es generar de manera permanente dinámicas de participación y trabajo conjunto con las familias y a la vez propuestas de actividades grupales integrando a toda la institución. Evidenciamos también como percepción de "buena práctica docente" el trabajo no centrado o exclusivo desde la currícula sino también pensando en sus necesidades más inmediatas, en su contexto, en sus particularidades o características individuales. Podemos considerar como uno de los indicadores de inclusión la matrícula aumentada de los niños comparando al inicio de esta gestión y en la actualidad, la ampliación del radio de influencia de las comunidades que asisten al jardín.

\section{Bibliografía}

Carli, S. (2012). Niñez, pedagogía y política: transformaciones de los discursos acerca de la infancia en la infancia de la educación argentina 1880-1955. 2da. ed. Buenos Aires. Miño y Dávila.

-(2012). (compil.) La cuestión de la infancia. Entre la escuela y el shopping. Buenos Aires. Paidós 

Editores.

Freire, P. (2009). El grito Manso. 2ºEdic. Buenos. Aires. Siglo Veintiuno.

Narodowski, M. (2008). La inclusión educativa. Reflexiones y propuestas entre las teorías, las demandas y los slogans. Revista Electrónica lberoamericana sobre Calidad, Eficacia y Cambio en Educación, 6, (2), 19- 26.

Oporto, M. L. 2015.Presentación curso de capacitación docente de www.wdu.ar Presentaciones- Gestiòn y uso didáctico- La Educaciòn de Gestiòn Social ) Disponible en

http://www.fediap.com.ar/administracion/pdfs/La\%20Educacion\%20de\%20Gestión\%20 Social\%20-\%20Prof.\%20Mónica\%200porto.pdf

Perrone, E. (2014). Módulo de capacitación del Postítulo de Pedagogía y Educación Social. Ministerio de Educación. Presidencia de la Nación Argentina. Buenos Aires. Universidad de Santiago de Compostela España.

Tapia, L. (2009). Movimientos sociales, movimientos societales y los no lugares de la política. Cuadernos del pensamiento crítico latinoamericano, 17, (2), 1-4 (en línea). http://www.jornada.unam.mx/2009/03/03/luis.pdf, acceso 30 de marzo de 2017.

UNICEF. (1989). Convención sobre los derechos del niño (en línea). https://www.unicef.org/argentina/spanish/7.- Convencionsobrelosderechos.pdf, acceso marzo de 2017.

Zabalza M. A. (2012). Calidad en la Educación Infantil. 3. Los diez aspectos claves de una Educación Infantil de calidad (en línea). http://www2.uned.es/489130/Textos/Tema12.pdf, acceso 10 de diciembre de 2016.

Zabalza Beraza, M. A. (2012). El estudio de las "buenas prácticas" docentes en la enseñanza universitaria. Revista de Docencia Universitaria, 10, (1), 17-42. (en línea). http://red-u.net/redu/documentos/vol10 n1 completo.pdf , acceso 15 de octubre de 2016.

\section{Notas}

i Cristina Cantero y su esposo, Adolfo "Fito" Molodezky trabajaron en el terreno social, desde la izquierda, su figura y la de su esposa crecieron en la conducción del MTL (1998-1999). El movimiento cobró gran visibilidad en 2002 con la ocupación de terrenos en un predio de Marconi y ruta 11, donde hoy funciona una reconocida cadena de supermercados. Molodesky fue docente egresado del Instituto Superior de Educación Técnica y Formación Profesional. Lideró por años movilizaciones y protestas gremiales (Agremiación de Trabajadores Docentes) durante los años noventa y después de la crisis institucional de 2002.Junto con Alberto Tito Tabares, defendía un modelo educativo diferente, más cercano al modelo comunitario de otras experiencias latinoamericanas que finalmente cobró forma en la escuela de gestión social "Héroes Latinoamericanos". Falleció en un trágico accidente en el año 2016.

ii THEOMAI N 22 segundo semestre 2010 second semester 2010 . La experiencia del MTD 17 de Julio en el Chaco: de la resistencia a la producción (2000-2006) Maximiliano E. Román. Disponible en http://revista-theomai.unq.edu.ar/NUMERO\%2022/Art_Roman.pdf 